\title{
Characterization of Gel16 as a Cytochrome P450 in Geldanamycin Biosynthesis and in-silico Analysis for an Endogenous Electron Transport System ${ }^{\mathrm{S}}$
}

\author{
Hemraj Rimal ${ }^{1 \dagger}$, Sang-Cheol $\mathrm{Yu}^{1 \dagger}$, Byeongsan Lee ${ }^{2}$, Young-Soo Hong ${ }^{2 *}$, and Tae-Jin $\mathrm{Oh}^{1,3,4}$ \\ ${ }^{1}$ Department of Life Science and Biochemical Engineering, Sun Moon University, Asan 31460, Republic of Korea \\ ${ }^{2}$ Chemical Biology Research Center, Korea Research Institute of Bioscience and Biotechnology, Ochang-eup, Chungbuk 28116, Republic of Korea \\ ${ }^{3}$ Genome-based BioIT Convergence Institute, Asan 31460, Republic of Korea \\ ${ }^{4}$ Department of Pharmaceutical Engineering and Biotechnology, Sun Moon University, Asan 31460, Republic of Korea
}

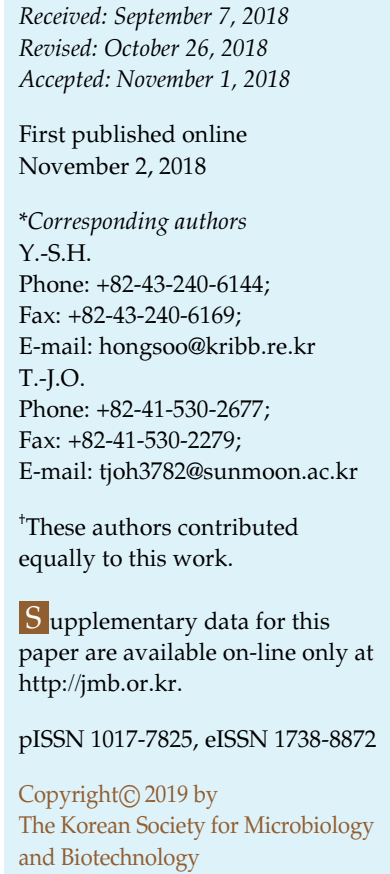

Geldanamycin and its derivatives, inhibitors of heat shock protein 90, are considered potent anticancer drugs, although their biosynthetic pathways have not yet been fully elucidated. The key step of conversion of 4,5-dihydrogeldanamycin to geldanamycin was expected to catalyze by a P450 monooxygenase, Gel16. The adequate bioconversions by cytochrome P450 mostly rely upon its interaction with redox partners. Several ferredoxin and ferredoxin reductases are available in the genome of certain organisms, but only a few suitable partners can operate in full efficiency. In this study, we have expressed cytochrome P450 gel16 in Escherichia coli and performed an in vitro assay using 4,5-dihydrogeldanamycin as a substrate. We demonstrated that the in silico method can be applicable for the efficient mining of convenient endogenous redox partners (9 ferredoxins and 6 ferredoxin reductases) against CYP Gel16 from Streptomyces hygroscopicus. The distances for ligand FDX4-FDR6 were found to be $9.384 \AA$. Similarly, the binding energy between Gel16-FDX4 and FDX4-FDR6 were $-611.88 \mathrm{kcal} / \mathrm{mol}$ and $-834.48 \mathrm{kcal} / \mathrm{mol}$, respectively, suggesting the lowest distance and binding energy rather than other redox partners. These findings suggest that the best redox partners of Gel16 could be NADPH $\rightarrow$ FDR6 $\rightarrow$ FDX4 $\rightarrow$ Gel16.

Keywords: Biosynthetic pathway, cytochrome P450, electron transport system, geldanamycin, in silico analysis, redox partner

\section{Introduction}

Cytochrome P450s (CYPs) are found in almost all the biological kingdoms and are heme-containing monooxygenases that catalyze a majority of drugs and foreign lipophilic compounds [1]. Bacterial CYPs also catalyze most reactions of environmental and medicinal importance. Among microorganisms, actinomycetes are responsible for producing a vast array of antibiotics and natural products that are valuable in human welfare [2]. Geldanamycin is an antitumor benzoquinone ansamycin produced by Streptomyces hygroscopicus. It is a well-known inhibitor of heat shock protein 90 (Hsp90) and possesses ATPase activity necessary for the maturation and activation of proteins that are responsible for carcinogenesis and tumor development [36]. Gel16 is a CYP monoxygenase that catalyzes the C4-C5 double-bond formation of 4,5-dihydrogeldanamycin as a final biosynthetic step in the geldanamycin biosynthetic pathway as shown in Fig. 1 [7-9]. The efficiency of bioconversion with CYP largely relies on its interaction with redox partners containing ferredoxin (FDX) and ferredoxin reductase (FDR) as well as within redox partners themselves. Among 9 FDXs and 6 FDRs from S. hygroscopicus genome (data not published), it is still unknown which of its real redox partners interacts with Gel16 for the optimum overproduction geldanamycin. 


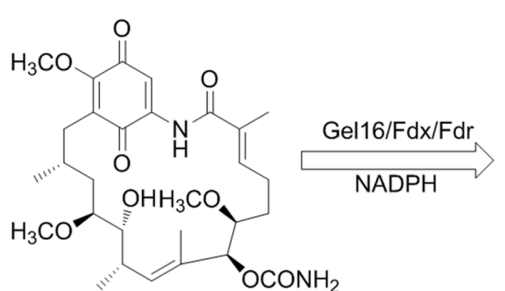

4,5-dihydrogeldanamycin

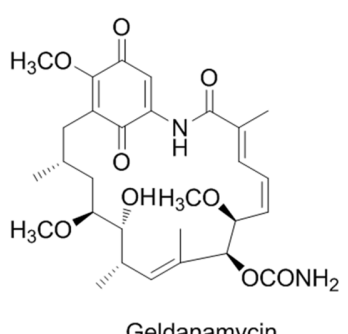

Geldanamycin
Fig. 1. Schematic representation of geldanamycin from 4,5dihydrogeldanamycin by CYP Gel16.

Gel16-mediated bioconversion of 4,5-dihydrogeldanamycin to geldanamycin was done by using FDX and FDR as electron transfer partners in which cofactor NADPH is oxidized to $\mathrm{NADP}^{+}$.

CYPs need auxiliary proteins to transfer electrons as they cannot receive directly from reduced pyridine nucleotides (exception CYP55A subfamily) [10]. Bacterial CYPs follow different mechanisms for the transportation of electrons. The basic rule for the flow of electrons is from $\mathrm{NAD}(\mathrm{P}) \mathrm{H}$ to the flavoproteins. Bacterial CYPs get electrons either from flavoproteins or from FDX, which in turn receives them from FDR [11]. Little study has been done on endogenous Streptomyces transport proteins like FDX and FDR [12].

In this study, we are able to express the gel16 gene, which has sequence similarity with 105 CYP subfamilies. Histagged Gel16 was overexpressed in E. coli and purified. Also, we confirmed that Gel16 can desaturate at the C-4 and $\mathrm{C}-5$ position of geldanamycin forming a double bond. The apparent dissociation constant (Kd), Michaelis constant $(\mathrm{Km})$ and maximum reaction rate (Vmax) values of Gel16 against 4,5-dihydrogeldanamycin were also determined. In addition, for the purpose of finding the real endogenous redox partner of Gel16, we performed an in silico study. We used protein-protein docking software, Z-dock and Rdock developed by Chen [13] and distributed by Accelrys [14]. Homology models of Gel16 along with FDRs and FDXs were made by using the Discovery Study (DS) 3.5. Besides these, protein-protein docking was done using ZDOCK and docked structure was refined by rDOCK. The interaction energy and distance between the docked proteins was calculated to find out the most stable docked protein complex. On the basis of these results, we can estimate the primary electron transport pathway of Gel16.

\section{Materials and Methods}

\section{Bacterial Strains, Plasmids and Growth Conditions}

Defined methods were applied for the isolation of total DNA, plasmid isolation and restriction digestion $[15,16]$. Luria-Bertani (LB) media provided with optimum amount of antibiotic was used for the growth of $E$. coli strains. The optimum growth temperature was $37^{\circ} \mathrm{C}$. Blue white screening was performed by using $0.4 \mathrm{mM}$ isopropyl- $\beta$-thiogalactopyranoside (IPTG) and $50 \mathrm{mM}$ 5-bromo-4chloro-3-indolyl- $\beta$-D-galactopyranoside (X-gal). PGEM-T Easy (Promega, USA) was used as cloning vector. pET28a(+) and pET32a $(+)$ (Novagen, USA) were used as expression vectors. In the same way, E. coli XL1-Blue MRF' (Stratagene, USA) and E. coli C41(DE3) were used as cloning and expression host, respectively.

Molecular Cloning, Over-Expression and Purification of Gel16

The codon optimization and synthesis of gel16 gene from S. hygroscopicus JCM4427 were performed using the GeneGPS program (DNA2.0). In order to construct an expression vector, the codon optimized Gel16 coding region was amplified with the primer opGel16-F (5'- CAT ATG GAC GAA ATT AGA GAT TAC CCA G - $\left.3^{\prime}\right)$ and opGel16-R (5' - CTC GAG TTA CCA CGC AAC $\left.C G-3^{\prime}\right)$. The correct gel16 gene was digested with the restriction enzyme (NdeI-XhoI) and ligated into pET-28a(+) to pET-opgel16. This plasmid, pET-opgel16, was transformed into E. coil C41(DE3), and the resulting strain was used for expression.

The selected colony as seed culture was inoculated in $6 \mathrm{ml} \mathrm{LB}$ broth containing $50 \mathrm{mg} / \mathrm{ml}$ kanamycin and grown at $37^{\circ} \mathrm{C}$ for a whole night. The seed culture was used for expression in 1-L volume. After the addition of seed culture, the cells were kept at $37^{\circ} \mathrm{C}$ at $150 \mathrm{rpm}$ until $\mathrm{OD}_{600}$ reached to 0.6. Then 1-mM 5- $\alpha$ aminoluvnic acid (ALA) and $0.5 \mathrm{mM}$ ferric chloride $\left(\mathrm{FeCl}_{3}\right)$ were added to the culture. After additional $15 \mathrm{~min}$ incubation at $20^{\circ} \mathrm{C}$, the protein expression was induced by $0.5 \mathrm{mM}$ IPTG and time duration for expression was $40 \mathrm{~h}$. The cells were collected by centrifugation and washed twice with $50 \mathrm{mM}$ Tris-HCl buffer $(\mathrm{pH}$ 7.5) containing $15 \%$ glycerol, $1 \mathrm{mM}$ DTT and $0.5 \mathrm{mM}$ EDTA. The cell pellets were mixed with $1.5 \mathrm{ml}$ of the same buffer and subjected to ultrasonication. After sonication, protein was subjected to centrifugation at 13,000 rpm for $30 \mathrm{~min}$ and a soluble portion was separated. The expression of redox partner PDX and PDR was done the same as in the previous work [17].

The soluble portion of the proteins was purified by using the immobilized metal affinity chromatography method. The proteins and talon resin were mixed in 10:1 ratio and were shaken for $1 \mathrm{~h}$. Then the proteins were centrifuged and washed by equilibrium potassium buffer at $\mathrm{pH}$ 7.5. The His-tagged proteins were washed with a double volume of $10 \mathrm{mM}$ imidazole followed by elution with $200 \mathrm{mM}$. The eluted proteins were concentrated with a Centricon filter (Merck Millipore, USA) and checked by SDSPAGE.

\section{Spectroscopic and Kinetic Assays}

The CO-reduction of His-tagged Gel16 was done by the following defined methods [18]. In this method, protein was diluted with $50 \mathrm{mM}$ potassium buffer containing 10\% glycerol. A pinch of sodium dithionate was added to and divided into two 
cuvettes, one as a reference and the other as sample. The sample cuvette was saturated with $40 \sim 50$ bubbles of carbon monoxide. Scanning was done between 400 and $500 \mathrm{~nm}$ at room temperature using a SHIMADZU 1601PC spectrophotometer. The CYP content was calculated by the difference in the absorbance values at $450 \mathrm{~nm}$ and $490 \mathrm{~nm}$, and an extinction coefficient of $91 \mathrm{~mm}^{-1} \mathrm{~cm}^{-1}$ was used.

Substrate binding assay was performed at room temperature after dilution of His-tagged Gel16 to $2.5 \mu \mathrm{M}$ in potassium phosphate buffer. Substrate concentrations ranging from $1 \mu \mathrm{M}$ to $300 \mu \mathrm{M}$ were mixed with protein and buffer. The absorbance values obtained $\Delta \mathrm{A}\left(\mathrm{A}_{\text {peak(390) }}-\mathrm{A}_{\text {trough(424) }}\right)$ were fitted to the MichaelisMenten model with GraphPad Prism 6.0 to calculate the dissociation constant. For determination of $\mathrm{Km}$ value, His-tagged Gel16 $(1 \mu \mathrm{M})$, spinach $\mathrm{Fdx}(10 \mu \mathrm{M})$, spinach Fdr $(3 \mu \mathrm{M})$ and 4,5-dihydrogeldanamycin were mixed in $50 \mathrm{mM}$ potassium buffer within $0.5 \mathrm{ml}$ total volume. The amount of substrate used ranged from $5 \sim 350 \mu \mathrm{M}$. After keeping it at $30^{\circ} \mathrm{C}$ for $5 \mathrm{~min}$, reaction was initiated by adding $300 \mu \mathrm{M}$ of NADPH, and measurement at $340 \mathrm{~nm}$ was taken by spectrophotometer. The NADPH consumption rate was calculated by an extinction coefficient of $6.22 \mathrm{mM}^{-1} \mathrm{~cm}^{-1}$ [19]. The NADPH consumption rate was converted into activity units (moles of NADPH oxidized per minute per mole of enzyme) and plotted against the different concentrations of 4, 5-dihydrogeldanamycin. The data were fitted to the Michaelis-Menten model using GraphPad Prism to obtain the tentative $\mathrm{Km}$ and Vmax values [20].

Enzymatic Bioconversion of 4,5-Dihydrogeldanamycin to Geldanamycin by His-Tagged Gel16

The in vitro assay was performed by using 4,5-dihydroxygeldanamycin as a substrate, $1 \mathrm{ml}$ of reaction mixture constitute of $1 \mu \mathrm{m}$ His-tagged Gel16, $10 \mu \mathrm{M}$ spinach FDX, $3 \mu \mathrm{M}$ spinach FDR, $5 \mathrm{mM}$ glucose-6-phosphate, $7 \mathrm{U}$ glucose-6-phosphate dehydrogenase, $100 \mu \mathrm{g} / \mathrm{ml}$ catalase, $10 \mathrm{mM} \mathrm{MgCl}_{2}, 300 \mu \mathrm{M}$ 4,5-dihydrogeldanamycin and $50 \mathrm{mM}$ potassium phosphate buffer. After mixing the above chemicals and reagents, the sample was kept for $5 \mathrm{~min}$ at room temperature. Reaction was initiated by adding $300 \mu \mathrm{M}$ of NADPH and was kept in $30^{\circ} \mathrm{C}$ for $2.5 \mathrm{~h}$. Reaction was stopped by adding double volume of ethyl acetate. After centrifugation, the upper portion was extracted and dried under nitrogen gas before methanol was used in further mixing.

\section{Analysis of Reaction Products}

Enzyme reactants were filtered through $0.2 \mu \mathrm{m}$ cellulose membrane syringe filters (Sartorius) and $20 \mu \mathrm{l}$ of samples were applied to a Kinetex biphenyl column $(250 \times 4.6 \mathrm{~mm}, 2.6 \mu \mathrm{m}$; Phenomenex, USA) using a U3000 HPLC system $(75 \% \mathrm{MeOH}$ for $25 \mathrm{~min}$ at isocratic flow rate of $1 \mathrm{ml} / \mathrm{min}$; Thermo Fisher Scientific, USA) equipped with a photodiode array detector. LC-MS was performed using a LTQ XL ion trap mass spectrometer (Thermo Fisher Scientific) equipped with an electrospray ionization (ESI) source.

\section{Construction of Homology Models and Docking 4,5- Dihydrogeldanamycin into Gel16}

The amino acid sequence of target protein Gel16 along with its redox partners and their homologous proteins were obtained from GenBank with accession numbers as shown in Table 1. The BLAST program against Protein Data Bank (PDB) available at NCBI was used to select template structure for homology modeling of all proteins. DS 3.5 version was used to construct the homology models [14, 21]. Heme coordinates for Gel16 were obtained with the well-known protein MoxA (2Z36), which already has a crystal structure and is positioned in the template [22]. By using CHARMM the structure was refined by minimization of the energy used for the molecular docking [23]. For the refined model, the Accelrys CHARMM force field was used for the simulation [24]. Validation of the homology models was done by ProSA 2003 z-score [25] and Ramachandran plot [24]. SuperPose was used for a statistical analysis of the template-based

Table 1. Average distance obtained using R-dock in the Gel16-FDXs, FDX4-FDRs and FDX8-FDRs.

\begin{tabular}{cccccc}
\hline Protein interaction & $\begin{array}{c}\text { Average distance } \\
(\AA)\end{array}$ & Protein interaction & $\begin{array}{c}\text { Average distance } \\
(\AA)\end{array}$ & $\begin{array}{c}\text { Protein interaction } \\
\text { Average distance } \\
(\AA)\end{array}$ & $\begin{array}{c}\text { } \\
\text { Gel16-FDX1 }\end{array}$ \\
9.611 & FDX4-FDR1 & 34.549 & FDX8-FDR1 & 46.182 \\
Gel16-FDX2 & 18.716 & FDX4-FDR2 & 152.730 & FDX8-FDR2 & 142.741 \\
Gel16-FDX3 & 20.063 & FDX4-FDR3 & 152.416 & FDX8-FDR3 & 142.411 \\
Gel16-FDX4 & $\mathbf{9 . 3 8 4}$ & FDX4-FDR4 & 195.905 & FDX8-FDR4 & 189.652 \\
Gel16-FDX5 & 20.093 & FDX4-FDR5 & 152.410 & FDX8-FDR5 & 142.407 \\
Gel16-FDX6 & 19.913 & FDX4-FDR6 & $\mathbf{1 2 . 0 3 2}$ & FDX8-FDR6 & $\mathbf{1 7 . 7 1 0}$ \\
Gel16-FDX7 & 20.037 & Spinach (FDX-FDR) & 12.370 & Spinach (FDX-FDR) & 12.370 \\
Gel16-FDX8 & $\mathbf{9 . 2 3 7}$ & PDX-PDR & 153.180 & & \\
Gel16-FDX9 & 19.693 & & & & \\
Gel16-FDX & 9.011 & & & & \\
(Spinach) & & & & & \\
Gel16-PDX & 10.384 & & & & \\
\hline
\end{tabular}


predicted model [26]. For drawing the substrate structure in the right stereo-chemically configuration a sketch tool bar application was used. That optimizes the structure by using the Dreiding-like force field in DS 3.5 along with LigandFit [27]. The optimized ligand molecule was docked into the precise Gel16 model and active sites of the enzyme were determined.

\section{Protein-Protein Docking by ZDOCK and Refining by rDock}

Using 9 FDXs and 6 FDRs obtained from the S. hygroscopicus genome, docking of Gel16-FDXs and FDXs-FDRs were done with ZDOCK, which is a rapid and basic stage algorithm for unbound rigid-body docking. Perfect docked poses were selected using Scores hits based on atomic contact energies, desolvation and electrostatic parameters [13]. The ZDOCK was conducted for Gel16-FDXs and FDXs-FDRs following the prescribed procedure to acquire only the top 2,000 ZDOCK poses within 60 clusters with distance cut-off of $6 \AA$ from the partner protein. After ZDOCK, clusters with a low pose Z-rank were selected. These clusters were further subjected to rDock, which is an easy and productive algorithm for refining unbound predictions created by ZDOCK. rDock is a three-stage energy minimization scheme, which evaluates electrostatic and desolvation energies. In the first two stages of minimization, ionic side chains were kept impartial, and reverted to their full charge states in the third stage of concise minimization [28].

\section{Interaction Energy Calculation between Gel16 and 6 FDXs}

The sum of the van der Waals and electrostatic energy of proteins is termed as 'interaction energy.' After fixed-backbone minimization of the docked complex, interaction energy was calculated. The minimization method consisted of a 20,000-step
Smart Minimizer minimization algorithm with an RMS gradient of 0.01 .

\section{Results}

\section{Gel16 Is a CYP Involved in C-4,5 Double Bond Formation of 4,5-Dihydrogeldanamycin}

The expressed and purified protein size of His-tagged Gel16 was 43.7 kDa (Fig. 2A). A cytosolic fraction obtained from the heterologous expression of His-tagged Gel16 showed a CO-reduced maximum difference at $450 \mathrm{~nm}$ (Fig. 2B). Similarly, protein concentration was found to be $186 \mu \mathrm{M}$.

As most of the bacterial CYPs are type-I class, the flow of electrons occurs from the $\mathrm{NAD}(\mathrm{P}) \mathrm{H}$ to the terminal CYP components known as auxiliary protein, viz. FDX and FDR $[29,30]$. Since Gel16 was a type-I CYP system, we used spinach FDX and spinach FDR as electron carrier partners to determine their function. As shown in Fig. 3A, the enzyme reactants with 4,5-dihydrogeldanamycin hit noticeable new peaks appearing at $15.3 \mathrm{~min}$ in the HPLC chromatograms, which was characterized as geldanamycin when compared with the spectra of a commercial standard. A parent mass ion peak was exhibited at $\mathrm{m} / \mathrm{z} 583.4[\mathrm{M}+\mathrm{Na}]^{+}$, which corresponded to dehydration of 4,5-dihydrogeldanamycin (a loss of 2 Da; Fig. S1). In the ESI-MS/MS spectrum, a daughter ion peak appeared at $\mathrm{m} / \mathrm{z} 187$ from geldanamycin and at 189 from 4,5-dihydrogeldanamycin in the Gel16 enzyme reactant, respectively. The formation of the $\mathrm{m} / \mathrm{z}$
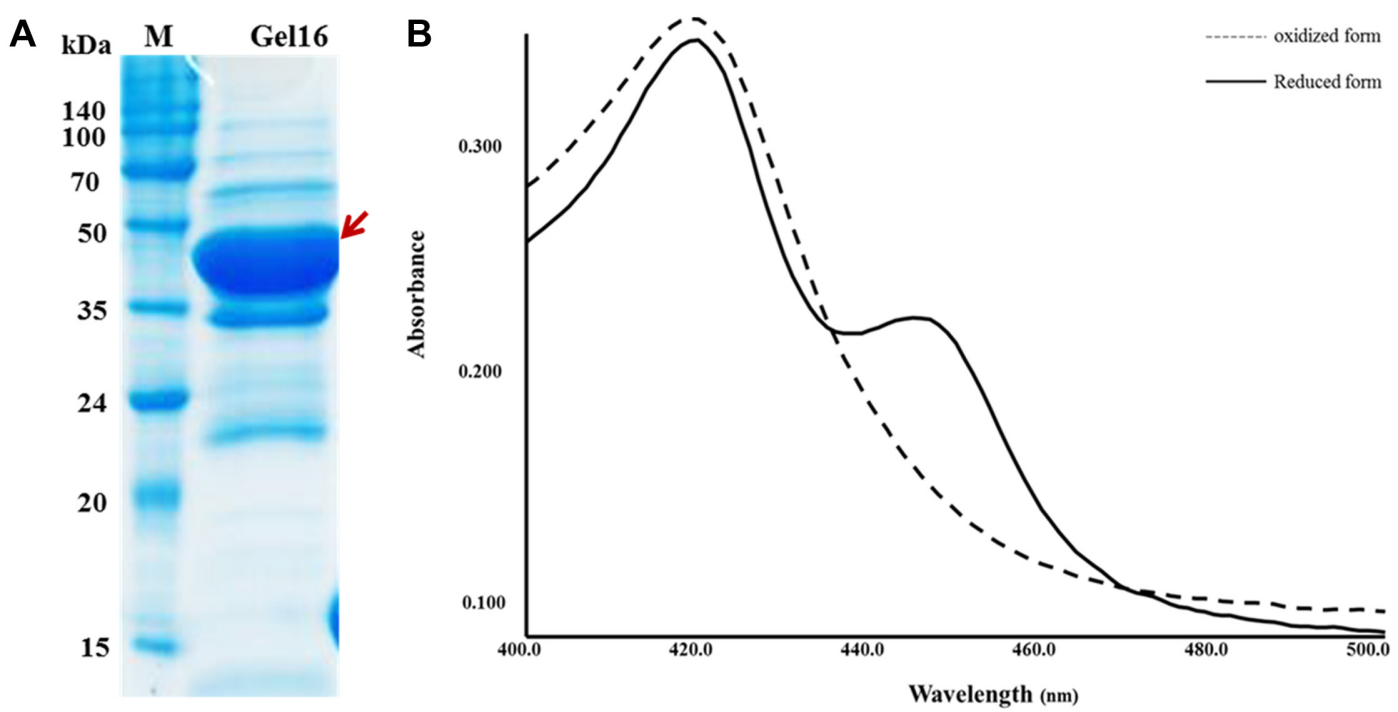

Fig. 2. Purification of His-tagged Gel16 and CO-binding assay.

(A) SDS-PAGE analysis of His-tagged Gel16. (B) CO-reduced spectra of His-tagged Gel16 where solid line is oxidized form and dotted line is COreduced form. 


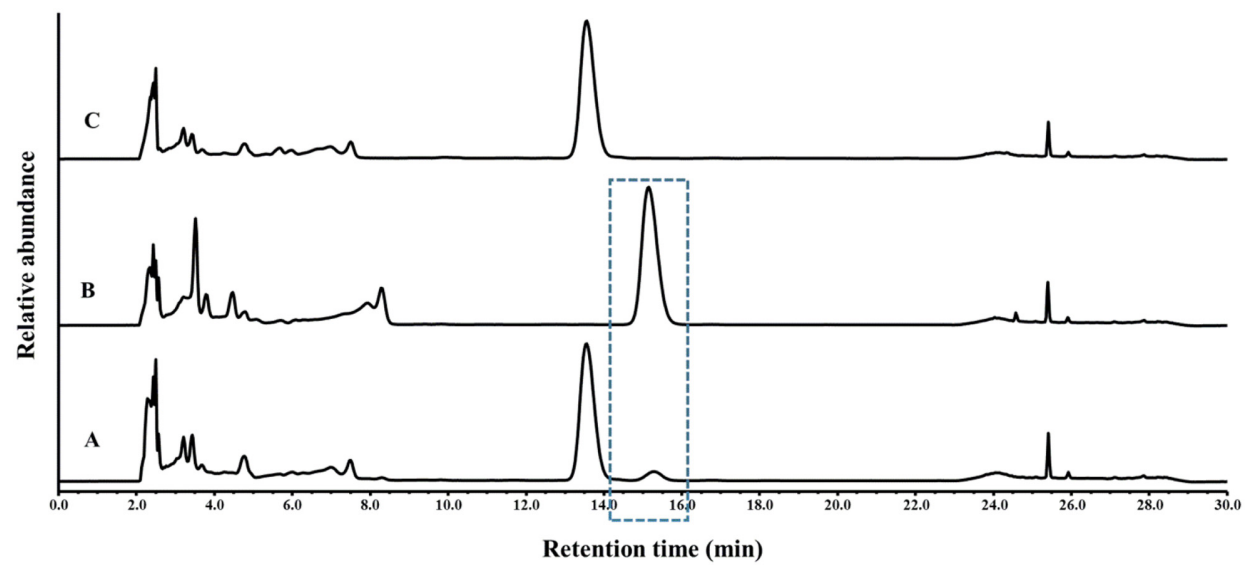

Fig. 3. In-vitro enzymatic conversion of 4,5-dihydroxygeldanamycin to geldanamycin.

HPLC profile of the in-vitro enzyme reaction mixture of the purified His-tagged Gel16 with 4,5-dihydrogeldanamycin (A), enzyme reaction mixture of the purified His-tagged Gel16 with geldanamycin (B), and control reaction mixture with 4,5-dihydrogeldanamycin without the purified His-tagged Gel16 (C). The peak at $15.3 \mathrm{~min}$ was found to correspond to geldanamycin.

187.0 ion commonly appeared for the C-4,5-saturated geldanamycin but not for the 4,5-dihydrogeldanamycin derivatives [31]. Its identity was confirmed as a geldanamycin (MW 560) from the MS/MS analysis of the $\mathrm{m} / \mathrm{z} 583.4[\mathrm{M}+\mathrm{Na}]^{+}$ion with daughter ions at $\mathrm{m} / \mathrm{z} 522$ and 187 (Fig. S1).

The substrate binding affinity of 4,5-dihydro-geldanamycin towards His-tagged Gel16 showed a Kd value of $1.5 \pm$ $0.1 \mu \mathrm{M}$ (Fig. 4A), denoting the strong binding affinity between our protein and substrate and also indicating the high capacity of the substrate to leave the receptor of Gel16. Moreover, the fitting of activity of His-tagged Gel16 against different concentrations of 4,5-dihydrogeldanamycin resulted in the hyperbolic curve in the Michalis-Menten model

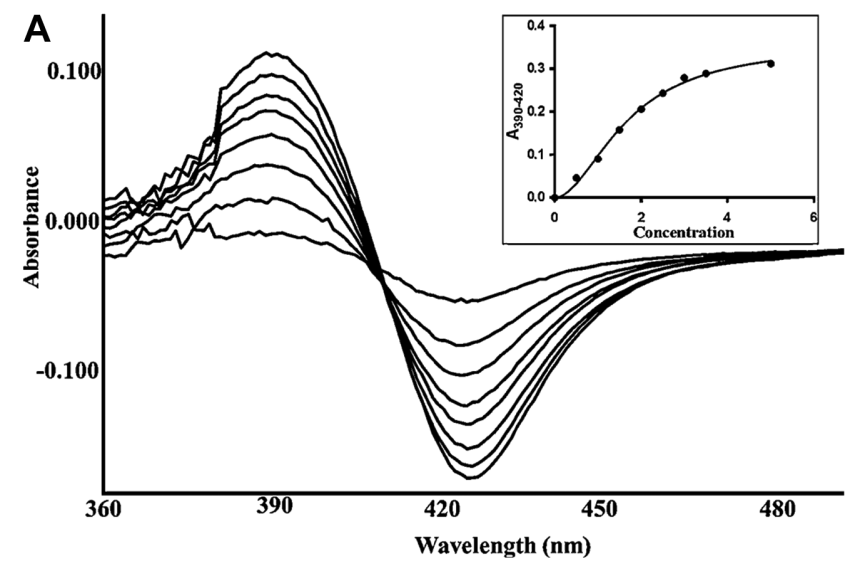

Fig. 4. Substrate binding affinity and the hyperbolic curve of Gel16.

(A) Substrate binding of Gel16 with 4,5-dihydrogeldanmycin. The insets show the hyperbolic curve after fitting $\Delta \mathrm{A}_{(390-420)}$ against the different concentrations of substrate. (B) Michaelis-Menten curve of Gel16 using 4,5-dihydrogeldanmycin as a substrate (10-350 $\mu \mathrm{M})$.

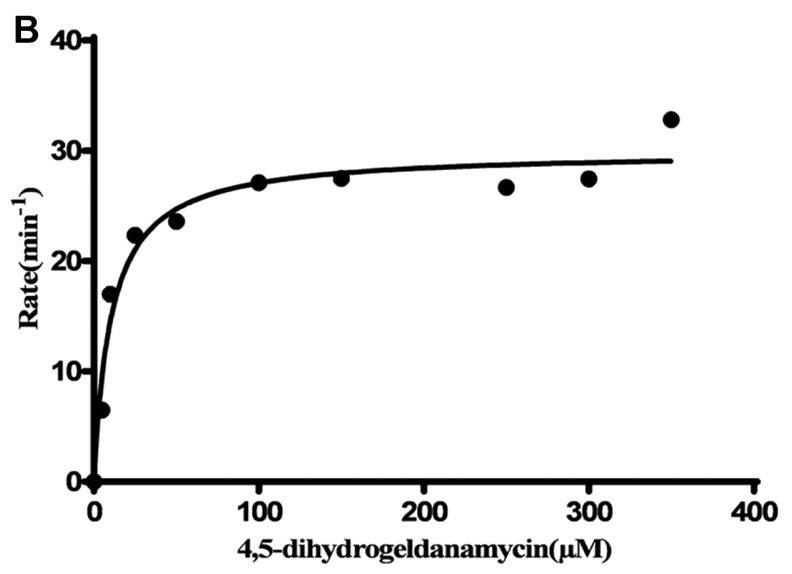

(Fig. 4B). The data obtain were $\mathrm{Km}$ value of $10.5 \pm 2.3 \mu \mathrm{M}$ and Vmax value of $29.9 \pm 1.0 \mathrm{~min}^{-1}$. The values of both $\mathrm{Km}$ and Vmax showed that Gel16 became saturated with a lower concentration of 4,5-dihydrogeldanamycin.

\section{Gel16 Showed the Lowest Energy Conformation with the Hydroxylation Sites of the 4,5-Dihydrogeldanamycin}

The target sequence and its template protein structure obtained from the PDB database has been summarized in Table S1. The structural templates with the most homologous sequences with respects to Gel16 were used for comparative homology modeling. The templates achieved through 2D alignment were an adaptable match with the Gel16 in case of both alignment and conserved residues containing helix, 
A
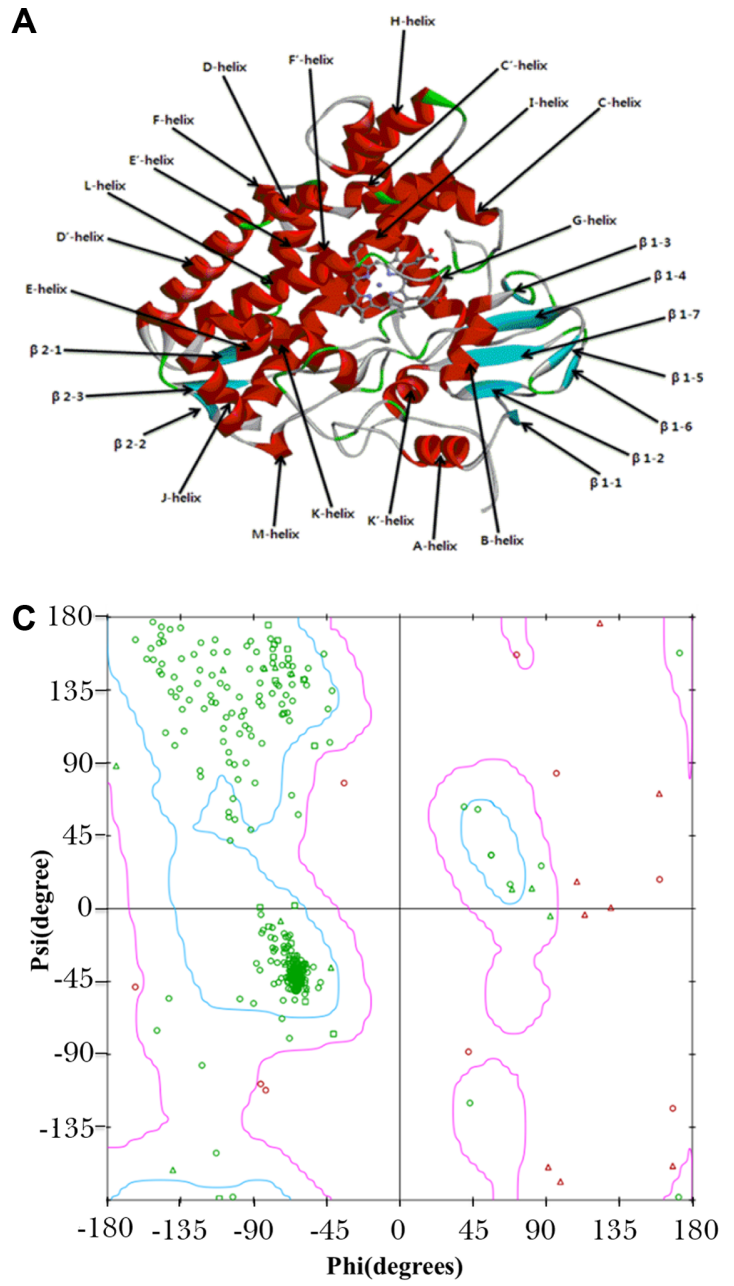

B

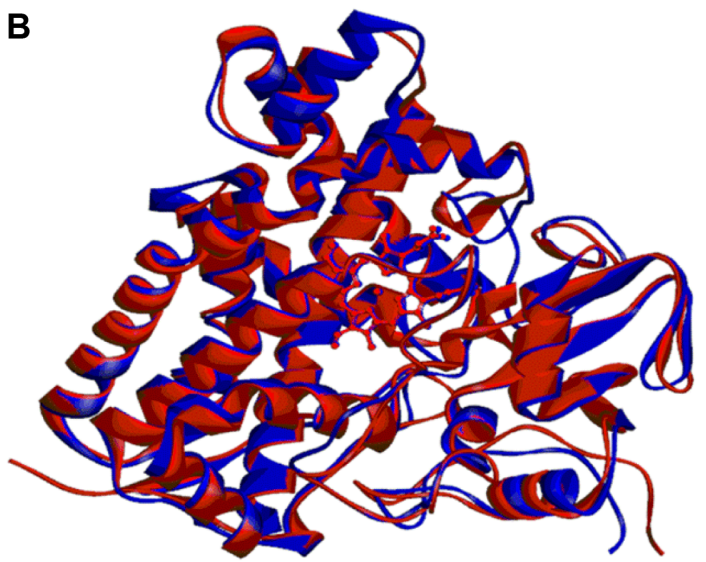

D

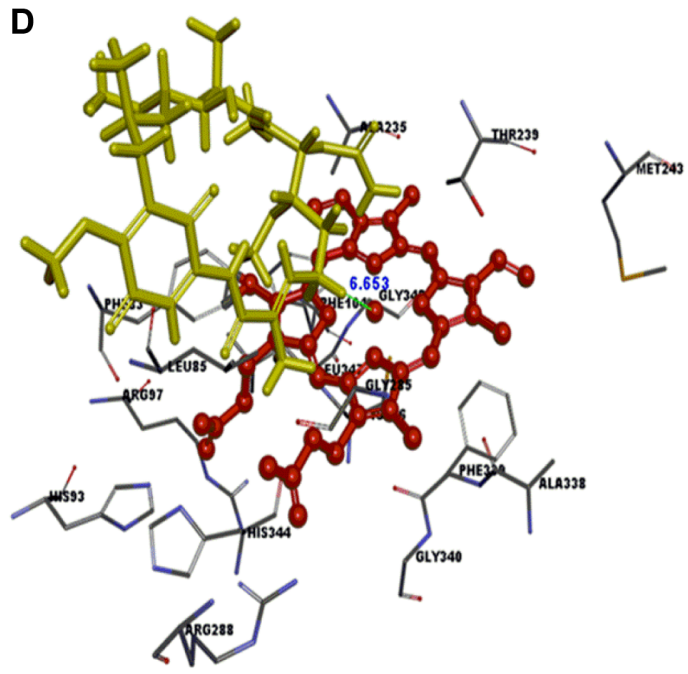

Fig. 5. Model validation and ligand docking.

(A) Structure of Gel16 model. $\alpha$ - Helices are in red; $\beta$-sheets are in blue; loops are in green and grey; and heme and porphyrin rings are in darkgrey. Conserved and important structures are labeled. (B) Superimposition of Gel16 model (blue) over (4UBS) (red). (C) Ramachandran plot of modeled structure of Gel16. (D) The ligands (4,5-dihydrogeldanamycin, yellow) are facing the heme within the active site of Gel16 (6.653 $̊$ ). The heme is shown as a red circle; the loops (grey) are drawn in stick representation; and the green line represents the distance between the heme and the carbon.

sheet and heme-binding motif (Fig. S2). Gel16 showed $45.5 \%, 44.1 \%$ and $43.6 \%$ sequence identity with $2 \mathrm{Z} 36,3 \mathrm{ABA}$ and $2 \mathrm{ZBX}$, respectively, and it was reasonable for model building. Gel16 conserved the various signature motifs. The structural motifs of Gel16 are pentapeptides I-helix (AxxLL), heptapeptides K-helix (AVxExxR) and L-helix $(x x x x \operatorname{Rx} \operatorname{Lx} x x x x x \mathrm{~L})$, and the characteristic signature hemebinding motif for the CYP subfamily is FxxGxHxCxGxxxxR [32]. The signature motif showed a conserved cysteine residue that ligated to the heme. These structural and characteristic reserve motifs verified the authenticity of the Gel16 model construction based on sequence alignments. In the same way, different FDXs and FDRs having maximum identity with already known proteins have been found. All FDXs showed a typical $\mathrm{C}_{x x} \mathrm{H}_{x x} \mathrm{C}\left(\mathrm{x}_{\mathrm{n}} \mathrm{CP}\right.$ ironsulfur cluster-binding motif [33]. FDX1 and FDX4 showed the maximum identity with $1 \mathrm{BC} 6$ with $35.5 \%$ and $40.7 \%$, respectively. Similarly, all other remaining FDXs showed maximum resemblance with the protein 1SJ1. Among six FDRs from S.hygroscopicus, FDR1 showed the highest resemblance with protein $4 \mathrm{H} 4 \mathrm{Y}$ whereas FDR2, FDR3 and FDR4 showed maximum identity with 3LXD. Likewise, FDR5 and FDR6 have more identity with 2GQW and 1LQT, respectively as shown in Table S1. Also, the structural NADPH binding motif of FDR6 is GVGNVAVDVA as shown in the general NADPH binding motif, GxGxxAxxxA 
$[34,35]$.

The 3D protein models were generated using Modeler Module in DS 3.5. In case of Gel16, the 3D structure of Gel16 was finally prepared (Fig. 5A), and backbone rootmean-square deviation (RMSD) for the model was obtained after superimposing Gel16 onto the most homologous template $2 Z 36$ from Nonomuraea recticatena (Fig. 5B). In addition, the Ramachandran-plot $(\phi / \Psi)$ distribution of the backbone conformation angles for each of the residues of the refined structure revealed that $89.7 \%, 5.0 \%$, and $5.3 \%$ were in the favored region, allowed region and outlier region, respectively (Fig. 5C). The same technique was followed for the model building and validation on the redox partners (Fig. S3 and S4). We supposed that our optimized models are acceptable and trustworthy for the remainder of the study.

The Gel16 model consists of helices A-L, ten beta sheets, and the connecting loops that link the two of them to each other. A comparison with other templates showed that our Gel16 model contains almost every fold and the following structurally conserved regions (SCR) [36]: helices E, I, K, and L. Gel16 also comprises structurally variable regions (SVR), which are also known as "plastic regions." The highly preserved and practically essential residues that are found mostly in CYPs also exist in Gel16; the consensus sequence FGxGxHxCxGxxLA in Gel16 contains the Cys responsible for heme binding. The 3D structure of Gel16 showed a large pocket just above the heme molecule that is believed to accommodate 4,5-dihydrogeldanamycin or other relatively large substrates. The ligand (4,5dihydrogeldanamycin) was docked into the active site using LigandFit, and residues in active sites were identified. The residues whose side-chain faces the substrate-binding pocket are Phe83, Leu85, His93, Arg97, Phe104, Ala235, Thr239, Met243, Gly285, Arg288, Ala338, Phe339, Gly340, His344, Cys346, Leu347 and Gly348 (Fig. 5D). It is worth noting that almost all of the residues involved in the binding of 4,5-dihydrogeldanamycin were located in the substrate recognition sites (SRSs) [37]. Our assumption with the hydroxylation sites of the 4,5-dihydrogeldanamycin allowed us to select the lowest energy conformation by considering the distance between the heme and substrate hydroxylation site with a high consensus score. We generated poses wherein $\mathrm{C} 4$ faced the heme with a distance of $6.653 \AA$ (Fig. 5D).

\section{Gel16-FDX4 and FDX4-FDR6 Are Best Combinations for Protein-Protein Docking}

The first step in protein-protein docking was performed by ZDOCK. A total of 60 clusters and 2,000 poses were generated (data not shown). The most consistent cluster, No. 1, which has the maximum number of favorable scoring poses as marked by ZRANK, was selected for refinement by rDOCK. Of all the poses in cluster No. 1, only the 5 top-scoring poses were made into a single group and further refined by rDOCK. The docked poses generated after ZDOCK were between Gel16-FDXs and FDXs-FDRs (data not shown). A multi-staged CHARMM energy minimization technique was used for determining the best complex in terms of energy. The E-RDock score calculated in terms of energy of the five poses was subjected to rDOCK refinement (data not shown). The ERdock molecular formula is: E_sol + beta*E_elec2, where "E_sol" is the desolvation energy of the protein complex and "E_elec2" are the electrostatic energies of the protein complex after successive CHARMM minimization.

On the basis of the rDOCK results, ligands were inserted from homology models made by DS 3.5, and their distance on the protein-protein models were measured. The average distance of 5 refined poses between Gel16-FDXs was found to match in the order of FDX8 > FDX4 > FDX1 with a short distance on Gel16 protein model. However, between the FDXs-FDRs, the average distance was found to be optimal among FDX4 and FDR6 with a much shorter distance than FDX8-FDR6 (Table 1). Therefore, we concluded that the best combinations are Gel16-FDX4 and FDX4-FDR6 by using their much shorter average distances between Gel16FDXs and FDXs-FDRs. In case of Gel16-FDX4, their protein-protein 3D model was finally prepared (Fig. 6A), and the distance measured between the Fe of Gel16 and FeS cluster of FDX4 was $9.384 \AA$ (Table 1 and Fig. 6B). The active site of Gel16-FDX4 showed some residues, whereby Leu56, Phe83, Asn84, Leu85, His93, Arg97, Val231, Leu232, Gly285, Thr286, Gly287, and Arg288 emerged from Gel16, and Cys25, Ile26, Tyr27, Glu39, Cys40, and Val41 emerged from FDX4 (Fig. 6B). Similarly, in the case of FDX4-FDR6, their protein-protein 3D model was finally prepared (Fig. 6C), and the distance measured between the Fe-S and FAD clusters was $12.032 \AA$ (Table 1 and Fig. 6D). The active site between FDX4-FDR6 shows that Thr2, His36, Pro37, Asp38, Glu39, Glu58, Lys66, Lys70, Gly83, Ser86, Lys87, Leu88, Gly89, Ile91, His95, Pro96, Leu101, Pro102, Pro103 and His106 emerged from FDX4, and Leu54, Gly58, Val59, Ala60, His63, Ile66, Val136, Asn164, Val165, Asp168, Tyr328, Arg370, Pro372, Gly374, Val375, Ile376, Gly377, and Asn379 emerged from FDR6 (Fig. 6D).

Similarly, the Gel16-FDX4 model proposed that the hydrogen bond interaction and hydrophobic contact 


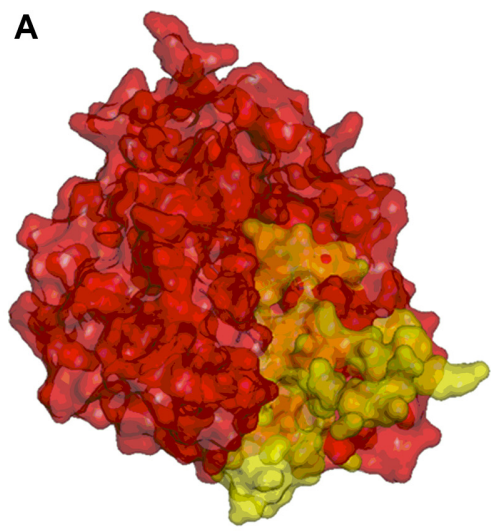

B
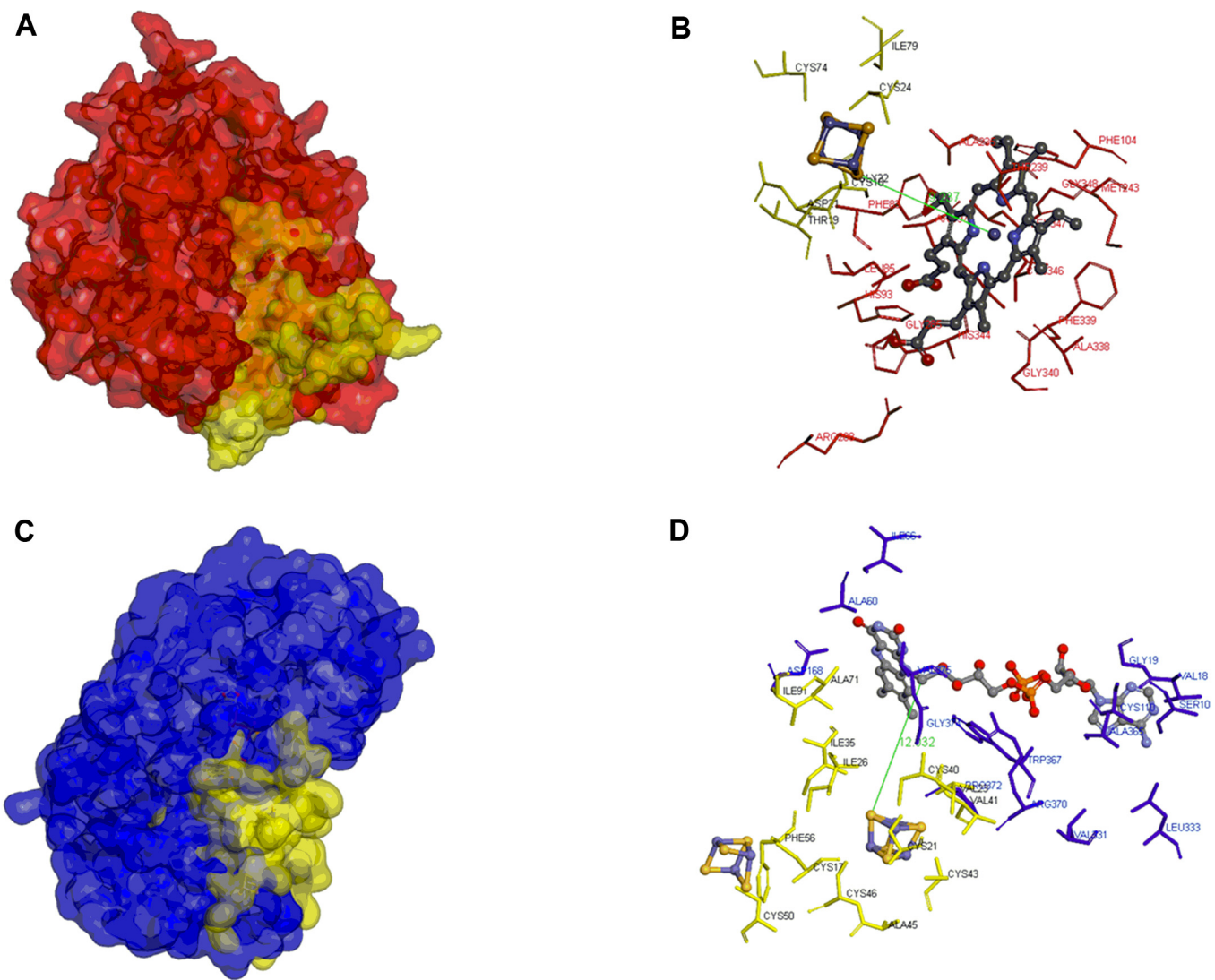

Fig. 6. The docking poses of proteins and binding sites in the Gel16-FDX4 and FDX4-FDR6.

(A) Surface model of Gel16-FDX4. (B) Residues between Gel16 and FDX4. (C) Surface model of FDX4-FDR6. (D) Residues between FDX4 and FDR6. Red, Gel16; yellow, FDX4; blue, FDR6; and heme is in blue/grey circle.

between Gel16 and FDX4, with an interface area of 1468.60 $\left(\AA^{2}\right)$, play crucial roles in their recognition and binding (Table S5). The interface area of Gel16-FDX4 and H-bonds is greater than that of other Ge16-FDXs combinations, thereby suggesting Gel16-FDX4 as the best combination.

\section{Calculation of Binding Energy between Gel16-FDXs and FDXs-FDRs Models}

The interaction energy between Gel16-FDXs and FDXsFDRs was calculated using CHARMM. The average interaction energy of 5 refined poses between Gel16-FDXs was found to be best with Gel16 and FDX4 with interaction energy of $-611.88485 \mathrm{kcal} / \mathrm{mol}$, and between FDXs-FDRs, the average was found to be optimal among FDX4 and FDR6 with interaction energy of $-834.48986 \mathrm{kcal} / \mathrm{mol}$ (Table 2). Heme (Fe) and 3Fe-4S were ignored during the binding energy calculation, for the reason that calculation of binding energy including ligands makes it unstable and assuming that protein binding energy is much stronger than the binding energy of the ligands inside a protein.

\section{Endogenous Redox Partners of Gel16}

According to the above mentioned, completely in silico

Table 2. Average binding energy obtained using rDOCK in the Gel16-FDXs and FDX4-FDRs.

\begin{tabular}{cccc}
\hline $\begin{array}{c}\text { Protein } \\
\text { interaction }\end{array}$ & $\begin{array}{c}\text { Binding energy } \\
(\mathrm{kcal} / \mathrm{mol})\end{array}$ & $\begin{array}{c}\text { Protein } \\
\text { interaction }\end{array}$ & $\begin{array}{c}\text { Binding energy } \\
(\mathrm{kcal} / \mathrm{mol})\end{array}$ \\
\hline Gel16-FDX1 & -580.64201 & FDX4-FDR1 & -764.45379 \\
Gel16-FDX2 & -226.09404 & FDX4-FDR2 & -477.52682 \\
Gel16-FDX3 & -259.93901 & FDX4-FDR3 & -139.22173 \\
Gel16-FDX4 & -611.88485 & FDX4-FDR4 & -323.78993 \\
Gel16-FDX5 & -386.17994 & FDX4-FDR5 & -300.03324 \\
Gel16-FDX6 & -362.19767 & FDX4-FDR6 & -834.48986 \\
Gel16-FDX7 & -304.34470 & Spinach (FDX-FDR) & -834.80394 \\
Gel16-FDX8 & -460.20690 & PDX-PDR & -314.08023 \\
Gel16-FDX9 & -285.12662 & & \\
Gel16-FDX & -578.31419 & & \\
(Spinach) & & & \\
Gel16-PDX & -336.41614 & & \\
\hline
\end{tabular}


analysis, we finally concluded that a more effective electron transport system could be achieved with the following: $\mathrm{NADPH} \rightarrow$ FDR6 $\rightarrow$ FDX $4 \rightarrow$ Gel16 (Fig. S3A). In addition, to confirm their possible protein combination, we prepared their total protein-protein structure between the Gel16FDX4 model, and the FDX4-FDR6 model (Fig. S3B), and measured the distance and their active sites in the total protein-protein combination structure, Gel16-FDX4-FDR6. Showing values befitting our expectations, the measured distances were $9.381 \AA$ and $12.106 \AA$ between Gel16 $(\mathrm{Fe})$ and FDX4 (Fe-S cluster), and between FDX4 (Fe-S cluster) and FDR6 (FAD cluster), respectively (Fig. S3C). Residues whose side-chain faces the protein-binding pocket are exactly the same as the active site of the Gel16-FDX4 and FDX4-FDR6 models as shown in Figs. 6B and 6D.

\section{Discussion}

Gel16, which plays a crucial role in the C-4,5 double bond formation to 4,5-dihydrogeldanamycin, has been previously studied using gene disruption and bioconversion experiments by our group $[7,9]$. It is clear that Gel16 is a last tailoring step enzyme which participates in double bond formation between C-4 and C-5 of 4,5-dihydrogeldanamycin in geldanamycin biosynthesis. There has been a lot of study related to geldanamycin biosynthesis and its derivatives for anti-cancer drug development. Most of the active and promising drug candidates in clinical trials, such as tanespimycin, alvespimycin, and IPI-493, are derivatives with a double bond on C-4 and 5 positions on the geldanamycin scaffold [38]. However, biochemical analysis of C-4,5 double bond formation and the Gel16-related redox partners that supply electrons to carry out the hydroxylation have not yet been competed and identified. Recently our group reserved the genome information of S. hygroscopicus and also confirmed the existence of 9 FDXs and 6 FDRs which can generate many possible combinations for an electron transport system for Gel16. These led us to our effort to identify and study the Gel16 redox partners.

Although many researchers have attempted to prepare soluble and stable CYP, FDX and FDR proteins from an E. coli heterologous over-expression system, it is so difficult and time consuming. Therefore, we used an in silico study using protein-protein docking between Gel16 and their electron transport-related proteins including FDXs and FDRs to investigate their possible combinations for electron transport chains. With the sequences of many a number of genomes revealed, comparative bioinformatics studies can be used to reveal protein interaction partners using several protein structures which have already been deposited in the PDB. Therefore, we carried out homology modeling of all of the protein containing Gel16 and redox partners and then applied the protein-protein interaction software DS 3.5. From a two-step docking analysis that consisted of ZDOCK and rDOCK (refinement by energy minimization), we finally observed rigid docking models.

The average distance between the refined models helped us to identify the best docked model structure. In case of Gel16 and FDXs, the best interaction distance was obtained for the Gel16-FDX8 and Gel16-FDX4 complex. Gel16-FDX8 $(9.237 \AA)$ has almost the same interaction distance with Gel16-FDX4 (9.384 $\AA$ ), and both are higher interaction energy than spinach FDX (9.011 ^), meaning that Gel16FDX8 and Gel16-FDX4 binds similarly weaker. However, in case of FDXs and FDRs, the best interaction distance was obtained for the FDX4-FDR6 complex. FDX4-FDR6 (12.032 Å) has lower interaction distance than FDX8-FDR6 (17.710 $)$ and spinach FDX-FDR (12.370 $)$, meaning that FDX4FDR6 binds stronger; therefore, Gel16-FDX4 and FDX4FDR6 are likely to have a more effective electron transport capacity in the Fe-S cluster and heme, and the Fe-S and FAD clusters, respectively.

The interaction between Gel16 with ferredoxin also supports a structural basis for ligand binding sites. We found that the Gel16-FDX4 complex has a bigger interface area than other Gel16-FDXs and showed more hydrophobic contacts. And it is known that protein-protein interactions are mostly driven by the hydrophobic effect. In addition, the average binding energy between the refined models helped us to identify the best docked model. In case of Gel16 and FDXs, the best interaction energy was obtained for the Gel16-FDX4 (-611.88485 kcal/mol) which has lower energy than spinach FDX $(-578.31419 \mathrm{kcal} / \mathrm{mol})$, meaning that Gel16-FDX4 binds more stably. Similarly in case of FDXs and FDRs, the best interaction energy was obtained from the FDX4-FDR6 $(-834.48986 \mathrm{kcal} / \mathrm{mol})$ which is almost the same value as spinach FDX-FDR $(-834.80394 \mathrm{kcal} / \mathrm{mol})$, meaning that both bind similarly. All of these in silico results led to the assumption that the electron transport in Gel16 carried out a reaction that was supplied in the following order: FDR6-FDX4-Gel16.

The study of protein-protein cooperation is at a climax. With the increasing number of important genomes exposed, use of comparative bioinformatics studies can be the best tool to report protein interaction partners. Deposition of fewer protein-protein complexes in PDB encouraged us to perform the in-silico method of protein-protein docking to 
find the best redox partner of Gel16 among many FDXs and FDRs from the genome of $S$. hygroscopicus. In order to find a suitable endogenous redox partner, methods like in-vitro reconstitution, gene disruption and quantitative reverse transcription polymerase chain reaction (qRT-PCR) mRNA and expression profile analysis etc., can be generally be performed [39], but are both time consuming and expensive. Thus, the over-expression of all redox partners both in soluble and active form is very tedious and almost impossible [40]. In the previous study we succeeded in finding favorable real redox partners of DoxA by in-silico study and validating it by in-vitro assay, by which we confirmed a 2-fold increase in production of doxorubicin from daunorubicin comparison with redox partners from spinach [41]. It was guaranteed that the protein-protein docking software ZDOCK and rDOCK can be applied for mining the best alternative redox partner in the class-I CYP with high confidence. Since protein-protein interaction determination by this method is reliable, so on the basis of these results we are going to clone the real redox partner for enzymatic assay of Gel16.

In-vitro assay of Gel16 was performed with 4,5-dihydrogeldanamycin, and reaction products that convert to geldanamycin were analyzed by HPLC and ESI MS/MS. This in silico study was also carried out to determine the best redox partner for better transfer of electron from cofactor to Gel16. Finally, we predicted electron transport in the following order: FDR6-FDX4-Gel16. Clues obtained from Gel16 model structure with active site architecture as well as the adjoining residues within substrate recognition site will be very beneficial for rational design of the enzymes for region selection and therefore to obtain more soluble and less toxic analogues of geldanamycin.

\section{Acknowledgments}

This research was supported by a grant (NRF2016R1D1A3B03933814) of the Basic Science Research Program through the National Research Foundation of Korea (NRF) funded by the Ministry of Education, Science and Technology, Republic of Korea. In addition, this work was supported by the Korea Polar Research Institute (grant no. PE18210) and the KRIBB Research Initiative Program.

\section{Conflict of Interest}

The authors have no financial conflicts of interest to declare.

\section{References}

1. Schenkman JB, Greim H, Zange M, Remmer H. 1969. On the problem of possible other forms of cytochrome P450 in liver microsomes. Biochim. Biophys. Acta 171: 23-31.

2. Chun YJ, Shimada T, Sanchez-Ponce R, Martin RV, Lei L, Zhao B, et al. 2007. Electron transport pathway for a Streptomyces cytochrome P450: cytochrome P450 105D5catalyzed fatty acid hydroxylation in Streptomyces coelicolor A3 (2). J. Biol. Chem. 282: 17486-17500.

3. BeBoer C, Dietz A. 1976. The description and antibiotic production of Streptomyces hygroscopicus var. geldanus. J. Antibiot. (Tokyo) 29: 1182-1188.

4. Johnson RD, Haber A, Rinehart KL Jr. 1974. Geldanamycin biosynthesis and carbon magnetic resonance. J. Am. Chem. Soc. 96: 3316-3317.

5. Prodromou C, Roe SM, O'Brien R, Ladbury JE, Piper PW, Pearl LH. 1997. Identification and structural characterization of the ATP/ADP-binding site in the Hsp90 molecular chaperone. Cell 90: 65-75.

6. Sasaki K, Rinehart KL Jr., Slomp G, Grostic MF, Olson EC. 1970. Geldanamycin. I. Structure assignment. J. Am. Chem. Soc. 92: 7591-7593.

7. Hong YS, Lee D, Kim W, Jeong JK, Kim CG, Sohng JK, et al. 2004. Inactivation of the carbamoyltransferase gene refines the post-polyketide synthase modification steps in the biosynthesis of the antitumor agent geldanamycin. J. Am. Chem. Soc. 126: 11142-11143.

8. Rascher A, Hu Z, Buchanan GO, Reid R, Hutchinson CR. 2005. Insights into the biosynthesis of the benzoquinone ansamycins geldanamycin and herbimycin, obtained by gene sequencing and disruption. Appl. Environ. Microbiol. 71: 4862-4871.

9. Shin JC, Na Z, Lee DH, Kim WC, Lee K, Shen YM, et al. 2008. Characterization of tailoring genes involved in the modification of geldanamycin polyketide in Streptomyces hygroscopicus JCM4427. J. Microbiol. Biotechnol. 18: 1101-1108.

10. Nakahara K, Tanimoto T, Hatano K, Usuda K, Shoun H. 1993. Cytochrome P-450 55A1 (P-450dNIR) acts as nitric oxide reductase employing $\mathrm{NADH}$ as the direct electron donor. J. Biol. Chem. 268: 8350-8355.

11. Paine M, Scrutton NS, Munro A, Gutierrez A, Roberts G, Wolf CR. 2005. Cytochrome P450: structure, mechanism, and biochemistry (Ortiz de Montellano, P. R., ed.). pp. 115148. 3rd Ed., Plenum Publishers New York.

12. O'Keefe DP, Gibson KJ, Emptage MH, Lenstra R, Romesser JA, Litle PJ, et al. 1991. Ferredoxins from two sulfonylurea herbicide monooxygenase systems in Streptomyces griseolus. Biochemistry 30: 447-455.

13. Chen R, Li L, Weng Z. 2003. ZDOCK: An Initial-Stage Protein-Docking Algorithm. Proteins 52: 80-87.

14. Discovery Studio 3.5. (2012) Accelrys Inc., San Diego, CA, 
USA. Available from: http://www.accelrys.com

15. Chang HC, Oriel P. 1994. Bioproduction of perillyl alcohol and related monoterpenes by isolates of Bacillus stearothermophilus. J. Food Sci. 59: 660-662.

16. Sambrook J, Russell DW. 2001. Molecular Cloning: A Laboratory Manual, 3rd Ed. Cold Spring Harbor Laboratory Press, Cold Spring Harbor, NY.

17. Bhattarai S, Liou K, Oh TJ. 2013. Hydroxylation of long chain fatty acids by CYP147F1, a new cytochrome P450 subfamily protein from Streptomyces peucetius. Arch. Biochem. Biophys. 539: 63-69.

18. Omura T, Sato R. 1964. The carbon monoxide-binding pigment of liver microsomes: Evidence for its hemoprotein nature. J. Biol. Chem. 239: 2370-2378.

19. Yang W, Bell SG, Wang H, Zhou W, Hoskins N, Dale A, et al. 2010. Molecular characterization of a class I P450 electron transfer system from Novosphingobium aromaticivorans DSM12444. J. Biol. Chem. 285: 27372-27384.

20. Gustafsson MC, Roitel O, Marshall KR, Noble MA, Chapman SK, Pessegueiro A, et al. 2004. Expression, purification, and characterization of Bacillus subtilis cytochromes P450 CYP102A2 and CYP102A3: flavocytochrome homologues of P450 BM3 from Bacillus megaterium. Biochem. 43: 5474-5487.

21. Baudry J, Rupasinghe S, Schuler MA. 2006. Class dependent sequence alignment strategy improves the structural and functional modelling of P450s. Protein Eng. Des. Sel. 19: 345353.

22. Yasutake $Y$, Imoto N, Fujii Y, Fujii T, Arisawa A, Tamura T. 2007. Crystal structure of cytochrome P450 MoxA from Nonomuraea recticatena (CYP105). Biochem. Biophys. Res. Commun. 361: 876-882.

23. Brooks BR, Bruccoleri RE, Olafson BD, States DJ, Swaminathan S, Karplus M. 1983. CHARMM: A program for macromolecular energy, minimization, and dynamics calculations. J. Comp. Chem. 4: 187-217.

24. Lovell SC, Davis IQ, Arendall III WB, De Bakker PI, Word JM, Prisant MG, et al. 2003. Structure validation by Calpha geometry: Phi, psi and C beta deviation. Proteins 50: 437-450.

25. Sippl MJ. 1993. Recognition of errors in three-dimensional structures of proteins. Proteins 17: 355-362.

26. Maiti R, Van Domselaar GH, Zhang H, Wishart DS. 2004. SuperPose: a simple server for sophisticated structural superposition. Nucleic Acids Res. 32: W590-W594.

27. Venkatachalam CM, Jiang X, Oldfield T, Waldman M. 2003. LigandFit: a novel method for the shape-directed rapid docking of ligands to protein active sites. J. Mol. Graph. Model. 21: 289-307.

28. Li L, Chen R, Weng Z. 2003. R DOCK: refinement of rigidbody protein docking predictions. Proteins 53: 693-707.
29. Guengerich FP. 1991. Reactions and significance of cytochrome P-450 enzymes. J. Biol. Chem. 266: 10019-10022.

30. Peterson JA, Lorence MC, Amarneh B. 1990. Putidaredoxin reductase and putidaredoxin: cloning, sequence determination, and heterologous expression of the proteins. J. Biol. Chem. 265: 6066-6073.

31. Lee JK, Jang JH, Park DJ, Kim CJ, Ahn JS, Hwang BY, et al. 2017. Identification of new geldanamycin derivatives from unexplored microbial culture extracts using a MS/MS library. J. Antibiot. 70: 323-327.

32. Rimal H, Yu SC, Jang JH, Oh TJ. 2015. Homology modeling and in vitro analysis for characterization of Streptomyces peucetius CYP157C4. J. Microbiol. Biotechnol. 25: 1417-1424.

33. Zhang T, Zhang A, Bell SG, Wong LL, Zhou W. 2014. The structure of a novel electron-transfer ferredoxin from Rhodopseudomonas palustris HaA2 which contains a histidine residue in its iron-sulfur cluster-binding motif. Acta Crystallogr. D. Biol. Crystallogr. 70: 1453-1464.

34. Karplus PA, Daniels MJ, Herriott JR. 1991. Atomic structure of ferredoxin-NADP ${ }^{+}$reductase: prototype for a structurally novel flavoenzyme family. Science 251: 60-66.

35. Lewis DF, Hlavica P. 2000. Interactions between redox partners in various cytochrome P450 systems: functional and structural aspects. Biochim. Biophys. Acta 1460: 353-374.

36. Hasemann CA, Kurumbail RG, Boddupalli SS, Peterson JA, Deisenhofer J. 1995. Structure and function of cytochromes P450: a comparative analysis of three crystal structures. Structure 3: 41-62.

37. Gotoh O. 1992. Substrate recognition sites in cytochrome P450 family 2 (CYP2) proteins inferred from comparative analyses of amino acid and coding nucleotide sequences. $J$. Biol. Chem. 267: 83-90.

38. Frankea J, Eichnera S, Zeilinger C, Kirschning A. 2013. Targeting heat-shock-protein 90 (Hsp90) by natural products: geldanamycin, a show case in cancer therapy. Nat. Prod. Rep. 30: 1299-1323.

39. Pandey BP, Choi KY, Lee N, Kim EJ, Kim JN, Yun H, et al. 2014. Identification of the specific electron transfer proteins, ferredoxin, and ferredoxin reductase, for CYP105D7 in Streptomyces avermitilis MA4680. Appl. Microbiol. Biotechnol. 98: 5009-5017.

40. Shrestha P, Oh TJ, Niraula NP, Liou K, Yoo JC, Sohng JK. 2010. Characterization of CYP166B1 and its electron transfer system in Streptomyces peucetius var. caesius ATCC 27952. Enzyme. Microb. Technol. 46: 372-377.

41. Rimal H, Lee SW, Lee JH, Oh TJ. 2015. Understanding of real alternative redox partner of Streptomyces peucetius DoxA: prediction and validation using in silico and in vitro analyses. Arch. Biochem. Biophys. 585: 64-74. 